\title{
TIME-DEPENDENT GATING IN NANOFLUIDIC CHANNELS FOR ACTIVE ELECTRIC DOUBLE LAYER MODULATION FOR ION TRANSPORT CONTROL
}

Caitlin Boone, Varun Lochab, Marie Fuest, and Shaurya Prakash ${ }^{*}$

Department of Mechanical and Aerospace Engineering

The Ohio State University, Columbus, Ohio, 43210, USA

\begin{abstract}
A nanofluidic field effect device was experimentally demonstrated with DC and time-dependent gated transport. Time-dependent transport was shown with sine and square waveforms over two orders of magnitude frequency range $(0.1-$ $10 \mathrm{~Hz}$ ) over four specific electrolyte concentrations in the $1 \times 10^{-4}$ $1 \times 10^{-1} \mathrm{M}$ range. Time dependent transport was found to have a higher degree of current modulation and a higher amount of total charge transfer through the nanofluidic channels compared to DC gating. An equivalent circuit model was developed to show the RC time constants for the PDMS, Stern, and diffuse layers which are hypothesized to be excited at the frequencies used in this experimental evaluation leading to enhanced ionic transport.
\end{abstract}

\section{INTRODUCTION}

Field effect nanofluidic devices (FENDs), geometrically analogous to solid-state field effect transistors, have been reported as diodes, rectifiers, and switches [1-3]. We report a FEND through a systematic study of sine and square wave gate modulation where, for the first time, time-dependent gating allows for active control over the electric double layer (EDL) for significant enhancement in measured current compared to purely DC gating at similar gate voltages. Time-dependent gating was selected for investigation the because introduction of time dependent signals are thought to require lower driving voltages than the DC counterpart [4] for equivalent device performance in microchannels. Moreover, we hypothesize that the active EDL modulation will permit enhanced total charge transfer through the nanochannels when applied frequencies are of similar magnitude to the native RC time constants for the EDL layers suggesting new technology for better pumping and ionic flow control. Technology development interest in FENDs arises from the operational length scales (sub-20 nm) at the level of fundamental biology with potential applications in water desalination [5], drug delivery [5], and DNA sequencing [2].

$\mathrm{AC}$ Electroosmosis (ACEO [6]) in microfluidics, relies on generating an effective electric field at a lower voltage than an equivalent $\mathrm{DC}$ signal by using an $\mathrm{AC}$ amplitude and frequency. The lower voltages limit faradaic reactions such as electrolysis and reduce Joule heating in microchannels. Moreover, as ACEO became better understood and the new name induced charge electroosmotic flow (ICEO) was proposed [7], it became evident that AC signals provides a component of the electric field arising from the induced charge to generate a body force on the ions and fluid. These ideas helped developed travelling wave electroosmotic pumps and separators [8,9] which have been useful in exploiting the non-linear electrokinetics in microfluidics due to use of AC signals.

It is worth noting that when a time-varying (i.e., AC) signal is used as a gate potential, at low frequencies (and hence the DC limit) nearly all the potential drop occurs across the EDL. In microfluidics, since most devices operate in a thin EDL regime ( $\kappa \mathrm{h}>>1$; where $\kappa$ is the inverse Debye length and $\mathrm{h}$ is the channel half-height) the transverse component of the electric field due to the AC potential at low frequencies $(1-10 \mathrm{~Hz})$ is too small to generate an appreciable body force and cause motion. At higher frequencies $\left(10^{3}-10^{5} \mathrm{~Hz}\right),[10,11]$ ACEO occurs and with increasing frequencies approaching the charge relaxation time limits the stable formation of the EDL. With a disrupted EDL, the space charge in the fluid near the wall is insufficient to generally induce net fluid motion [12]. Therefore, in microfluidics the 'sweet spot' for operating ACEO is in few $\mathrm{kHz}$ frequency ranges at voltages in the few $\mathrm{V}$ range $(<10 \mathrm{~V})$. [10, 11] Nanofluidics presents a different aspect and provides a new avenue to exploit surface charge for a new generation of fluidic platforms as opposed to the traditionally studies ACEO flow seen in microchannels.

At the nanoscale, with critical device dimensions below $100 \mathrm{~nm}$ (here at $16 \mathrm{~nm}$ ), $\kappa \mathrm{h} \leq 1$ or $\kappa \mathrm{h}<<1$, depending on electrolyte concentrations. Under such conditions, a significant portion of the nanochannel is occupied by the EDL. [13, 14] Therefore, at low frequencies, it is expected that an AC signal cause ion and fluid motion leading to quantifiable changes in measured current. Therefore, not only do we expect a much higher modulation of the field effect compared to a microchannel, we also expect a much broader range of frequencies to manipulate charge and mass transfer through the nanochannels while gaining in reduced joule heating and faradaic reactions, even with a single gate electrode instead of the array of gate electrodes seen in microchannels.

In microchannels, where almost all experimental work examining $\mathrm{AC}$ driven flow has been focused to date, direct influence of wall surface charge is limited in controlling bulk fluid flow. However, the use of multiple electrodes with high gating voltages $(\sim 10-100 \mathrm{~V})$ for velocity control [6] led to development of AC electroosmotic pumps. Additionally, a velocity dependence on frequency has been experimentally shown in microfluidic channels [10]. Yet, for nanochannels, frequency dependent gating is nearly non-existent with only two other limited experimental studies on frequency dependent fluid velocity $[4,15]$ and our previous report on measured current dependence on frequency $(0.1-0.5 \mathrm{~Hz})$ for a sine waveform and concentration. [16]

\section{METHODS}

Here, for the first time, we systematically investigated over a 2 orders of magnitude frequency range $(0.1-10 \mathrm{~Hz})$ for both sine and square gating, a RMS matched comparison against DC gating for aqueous $\mathrm{KCl}\left(1 \times 10^{-5}-0.1 \mathrm{M}\right)$ in $16 \mathrm{~nm}$ deep nanochannels. The FEND comprises a bank of three $2.5 \mathrm{~mm}$ long, $30 \mu \mathrm{m}$ wide, and $16 \mathrm{~nm}$ deep nanochannels connected to two microfluidic channel reservoirs $3 \mathrm{~cm}$ long, $50 \mu \mathrm{m}$ wide, and $8 \mu \mathrm{m}$ deep. The channel network was wet-etched into a borosilicate substrate with a gold gate electrode embedded in the nanochannel 'roof' and insulated fluidically and electrically by a polydimethylsiloxane (PDMS) layer (Figure 1). The entire device assembly was bonded with an $\mathrm{O}_{2}$ plasma bonding process. Fabrication methods for these long, ultra-low aspect ratio (ULAR) nanochannels has been reported by us previously $[3,16]$. Channels were filled with aqueous $\mathrm{KCl}\left(1 \times 10^{-5}-0.1 \mathrm{M}\right)$ at $\mathrm{pH} 7 \pm 0.02$. Independent voltage sources were used to generate the axial $\left(V_{a}\right)$ and gate $\left(V_{g}\right)$ 
potentials with current measured through a Keithley 6485 picoammeter. All testing equipment shared a common ground.

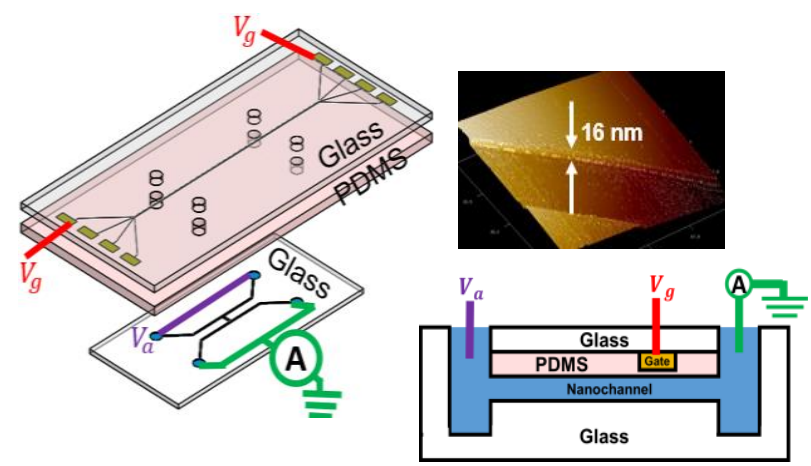

Figure 1: (Left) An exploded device schematic showing the three layer structure and locations of applied potentials and measured current. Only one of the 4 shown gate electrodes was active. (Top Right) a nanochannel AFM scan showing the $16 \pm 3 \mathrm{~nm}$ depth of the nanochannel. (Bottom Right) A cross sectional schematic view of the device. $V_{a}$ (purple) is the axial current driven through the nanochannels while $V_{g}(r e d)$ is applied signal to the gate electrode insulated from the nanochannels by the PDMS dielectric layer.

\section{RESULTS}

After verifying device operation $[3,16]$, the AC conductance using the $I_{R M S}$ (RMS current) from sine wave applied axially at $0.1 \mathrm{~Hz}$ showed the nanochannel conductance was higher than the purely DC axial signal for dilute electrolytes $(\leq 1 \mathrm{mM})$ or in the surface charge governed regime (SCGR) [2] (Figure 2). Notably, this result is in agreement with our hypothesis that at low frequencies, electroosmotic flow will occur and be more modulated than the equivalent (RMS voltage matched) DC counterpart since the total electric field is expected to be much higher in the transverse direction. The nanochannel conductance was to estimate the nanochannel depth to be $\sim 19 \mathrm{~nm}[3,5]$ in reasonable agreement with the etched channel depth of $16 \pm 3 \mathrm{~nm}$.

A representative plot of current vs. time for $V_{g}= \pm 2 \mathrm{~V} \mathrm{DC}$ and $V_{g}=2 \mathrm{~V}_{\mathrm{RMS}}$ for $\mathrm{AC}$ with axial potential $V_{a}=3 \mathrm{~V}$ is shown in Figure 3. $I_{R M S}$ was $66 \mathrm{pA}$ for sine gating, $73 \mathrm{pA}$ for square, and $I_{D C}$ was $6.3 \mathrm{pA}$ i.e., RMS matched AC provides nearly an order of magnitude higher measured current than DC. The rapid decay for the square wave (Figure 3B, shown with orange arrow) suggests the charging and discharging of the fluidic capacitors at the dielectric-fluid interface and subsequent control over the EDL. Since the flow is driven electrokinetically and is governed by ion transport, the measurement current over time was used to estimate the total charge, $Q$ that has passed through the nanochannel. Therefore, the area under a current-time plot (i.e., $\left.Q=\int_{0}^{T} I d t\right)$ where, $I$ is the current and $T$ is the measurement time.

For all cases in this work, a given gate voltage was applied over a 30 second period before the voltage was stepped up or down resulting in total charge values that can be directly compared (Figure 4).

Figure 4 shows $Q$ for all three tested frequencies $(0.1,1$, and $10 \mathrm{~Hz}$ ) across the 2 orders of magnitude range for both the sine and square wave. $Q$ increases with frequency for all measured concentrations in a sine wave while for a square wave gate input, the $1 \mathrm{~Hz}$ frequency shows the highest $Q$ in the square wave case, the $10 \mathrm{~Hz}$ signal that produces the highest current response in the

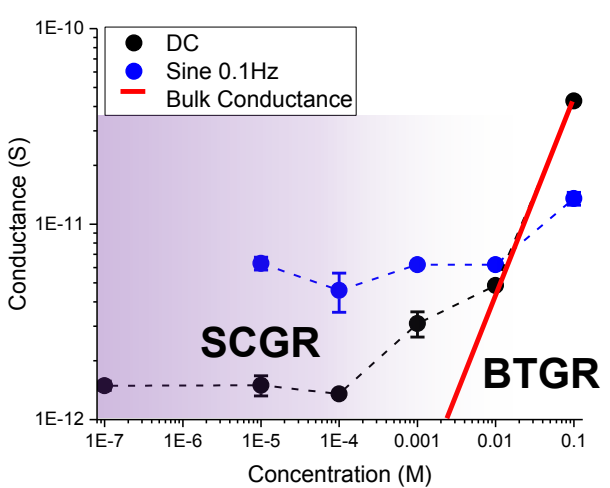

Figure 2: The black DC data points show device conductance in the surface charge governed (purple shading) and bulk transport governed regimes (no shading) [3]. The blue data shows nanochannel condutance using the $I_{R M S}$ for a $0.1 \mathrm{~Hz}$ sine wave. The red line is the bulk fluid conductance for a $19 \mathrm{~nm}$ channel which was seen to be in reasonable agreement with measured data. Dashed lines are provided as eye guides.

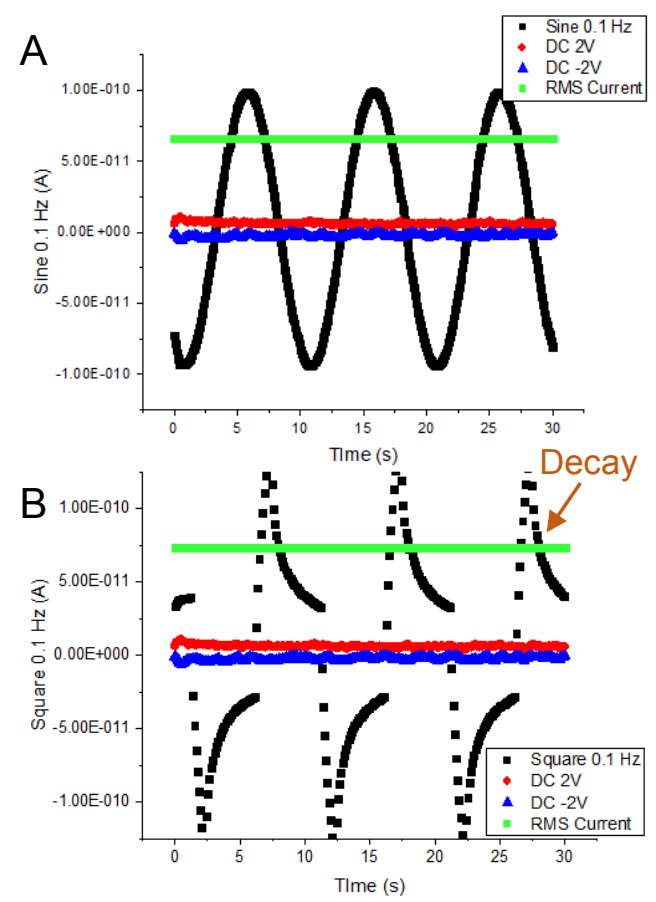

Figure 3: Current vs. time for the DC and $0.1 \mathrm{~Hz} A C$ cases at $1 \times 10^{-4} \mathrm{M}$ as a representative plot with $V_{a}=3 \mathrm{~V}$. (A) Sine waveform at the gate. (B) Square waveform at the gate. The plots show the charging and discharging cycle evident for square wave gating and also the increase in RMS current compared to DC only for similar gate voltage.

sine case shows the lowest charge transfer indicating an optimal frequency for charge transfer in the $0.1-10 \mathrm{~Hz}$ range, but requires further investigation. Additionally, in the sine wave cases, the total charge through the device remains relatively constant across frequency (with a slight drop moving from the SCGR to the BTGR) while in the square wave case, the charge transfer in the SCGR is constant but drops off in the BTGR. The differences in transport (both between frequencies and between 

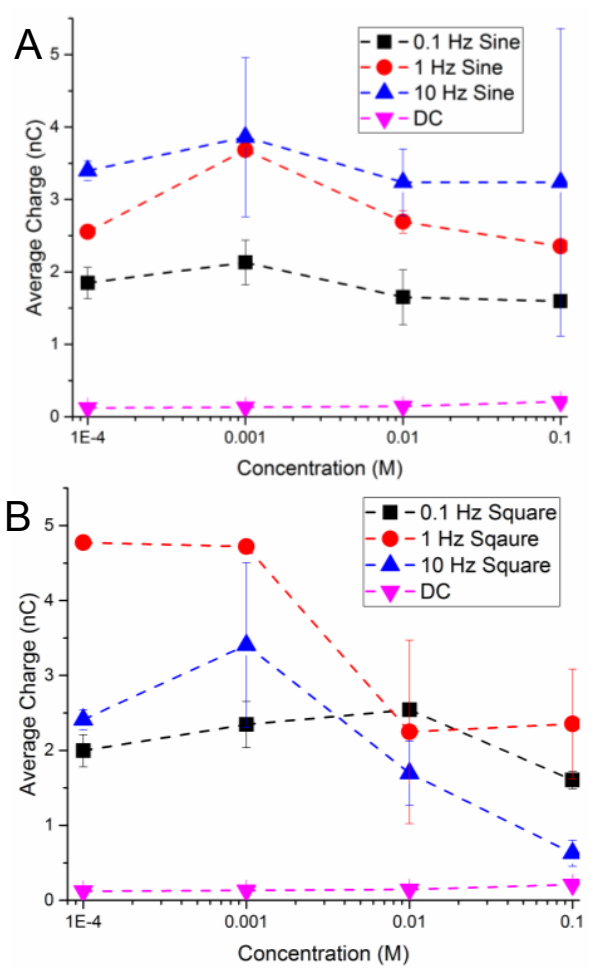

Figure 4: Average charge flowing through the nanochannels for both (A) Sine and (B) Square waveforms. $V_{a}=3 \mathrm{~V}$ in all cases. Dashed lines are eye guides. The pink data shows comparative $D C$ charge transfer. For sine gating, charge transfer increases with frequency and is relatively invariant across concentrations. But, for square wave gating, both a frequency and concentration dependence was seen, with charge transfer decreasing with increasing concentration.

$\mathrm{AC}$ and DC signals) decrease as the concentration increases and moved into the BTGR. All AC cases were shown to have higher average charge than the RMS matched DC case where charge through the device for the $\mathrm{V}_{\mathrm{g}}=2 \mathrm{~V}$ case was added to the $\mathrm{V}_{\mathrm{g}}=-2 \mathrm{~V}$ case.

A FEND equivalent circuit model was developed (Figure 5) for fitting to the measured current data. The model assigns a series of R/C circuit elements to the PDMS isolation layer, Stern layer, and the fluid diffuse layer and also accounts for the bulk fluid resistance. Each layer was modeled as a capacitor and resistor in parallel to allow for varying $\mathrm{RC}$ time constants for each component. The model solves the series of RC elements assigned to the three layers seen in Figure 5 for the total impedance according to the expression,

$$
\mathrm{Z}_{\mathrm{T}}=\frac{\mathrm{R}_{\mathrm{PDMS}}}{1+\mathrm{j} \omega \mathrm{C}_{\mathrm{PDMS}} \mathrm{R}_{\mathrm{PDMS}}}+\frac{\mathrm{R}_{\mathrm{S}}}{1+\mathrm{j} \omega \mathrm{C}_{\mathrm{S}} \mathrm{R}_{\mathrm{S}}}+\frac{\mathrm{R}_{\mathrm{D}}}{1+\mathrm{j} \omega \mathrm{C}_{\mathrm{D}} \mathrm{R}_{\mathrm{D}}}+\mathrm{R}_{\mathrm{b}}
$$

where $Z_{T}$ is the total impedance, $R_{P D M S}$ is the resistance of the PDMS, $R_{S}$ is the resistance of the Stern layer, $R_{D}$ is the resistance of the diffuse layer, $R_{b}$ is the bulk resistance, $\omega$ is the frequency of the applied signal, $\mathrm{C}_{\text {PDMS }}$ is the capacitance of the PDMS layer, $\mathrm{C}_{\mathrm{S}}$ is the capacitance of the Stern layer, and $\mathrm{C}_{\mathrm{D}}$ is the capacitance of the diffuse layer.

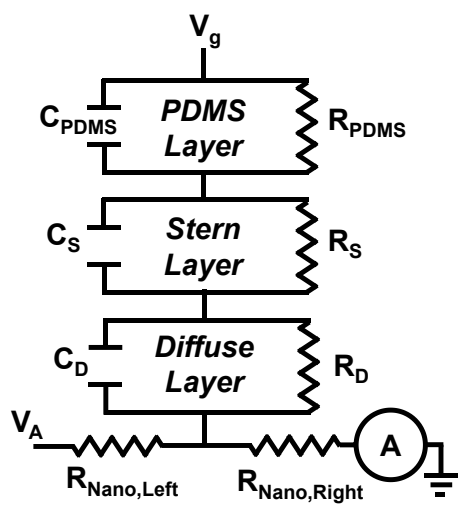

Figure 5: An equivalent circuit model for sine gating as a representative case. PDMS, Stern, and diffuse layers were modeled as $R / C$ circuits.

Since the $\mathrm{R}$ and $\mathrm{C}$ values were assumed to stay constant for a given electrolyte concentration, the bulk resistance value for each concentration (found from the DC conductance data in Figure 2) was also held constant. The model shows RC time constants for the PDMS layer was $\sim 97 \mathrm{~s}$ and diffuse layer was $\sim 0.02 \mathrm{~s}$ with all $\mathrm{RC}$ constants shown in Table 1 . Therefore, the gate voltage frequency can modulate diffuse layer ion distributions. Moreover, the Stern layer modulation would require frequencies on the order of $100 \mathrm{kHz}$ (based on $\mathrm{RC}$ time constants) so for the frequencies studied here, it would remain relatively in the steady state. The stability of the Stern layer throughout this process supports the claims this term can often be neglected when modeling similar systems. [17]

Table 1: A summary of the time constants from the Equivalent Circuit Model. Across three concentrations examined for each of the layers, the RC time constant was evaluated. The PDMS time constant $\left(R_{p d m s} C_{p d m s}\right)$ remained constant with concentration change, the Stern Layer time constant $\left(R_{S} C_{s}\right)$ remained constant with concentration change, and the diffuse layer time constant $\left(R_{d} C_{d}\right)$ changed with concentration as the layer thickness was altered. The model and data agree within 3\% and is well within the experimental error

\begin{tabular}{|l|c|c|c|}
\cline { 2 - 4 } \multicolumn{1}{c|}{} & \multicolumn{3}{c|}{ Time Constant (s) } \\
\cline { 2 - 4 } \multicolumn{1}{c|}{} & $1^{*} 10^{-4} \mathrm{M}$ & $1^{*} 10^{-3} \mathrm{M}$ & $1^{*} 10^{-2} \mathrm{M}$ \\
\hline $\mathrm{R}_{\mathrm{pdms}} \mathrm{C}_{\text {pdms }}$ & 97 & 97 & 97 \\
\hline $\mathrm{R}_{\mathrm{s}} \mathrm{C}_{\mathrm{s}}$ & $7.5 \mathrm{E}-5$ & $7.5 \mathrm{E}-5$ & $7.5 \mathrm{E}-5$ \\
\hline $\mathrm{R}_{\mathrm{d}} \mathrm{C}_{\mathrm{d}}$ & 0.021 & 0.019 & 0.026 \\
\hline
\end{tabular}

\section{CONCLUSIONS}

An AC gated FEND was demonstrated with two applied waveforms over a range of frequencies $(0.1-10 \mathrm{~Hz})$. AC gated FENDs were shown to have a more than an order of magnitude higher of charge transfer than the equivalent DC system while in the surface charge governed regime. An equivalent circuit model shows RC time constants for the PDMS layer was $\sim 97 \mathrm{~s}$ and diffuse layer was $\sim 0.02 \mathrm{~s}$. Therefore, the gate voltage frequency appear to modulate diffuse layer ion distributions based on the choice of gate frequencies used here. Moreover, the Stern layer modulation would require frequencies on the order of $100 \mathrm{kHz}$ and does not currently appear to be affected by our current gating platform. 


\section{ACKNOWLEDGEMENTS}

The authors would like to thank the staff of Nanotech West for assistance and training with equipment required for fabrication. We acknowledge partial financial support from NSF through grant number CBET-1335946 and NSF graduate fellowship program to support M. Fuest.

\section{REFERENCES}

1. Guan, W. and M.A. Reed, Electric Field Modulation of the Membrane Potential in Solid-State Ion Channels. Nano letters, 2012. 12(12): p. 6441-6447.

2. Karnik, R., et al., Electrostatic Control of Ions and Molecules in Nanofluidic Transistors. Nano letters, 2005. 5(5): p. 943-948.

3. $\quad$ Fuest, M., et al., A Three-State Nanofluidic FIeld Effect Switch. Nano letters, 2015. 15(4): p. 2365-2371.

4. van den Beld, W.T.E., et al. FREQUENCY DEPENDENT AC ELECTROOSMOTIC FLOW IN NANOCHANNELS. in Technical Digest of the 27th IEEE International Conference on Micro Electro Mechanical Systems, MEMS 2014. 2014. San Francisco, CA, United States: IEEE.

5. Guan, W., R. Fan, and M.A. Reed, Field-effect reconfigurable nanofluidic ionic diodes. Nature Communications 2011. 2.

6. Ramos, A., et al., AC Electric-Field-Induced Fluid Flow in Microelectrodes. Journal of Colloid Interface Science, 1999. 2(217): p. 420-422.

7. Bazant, M.Z. and T.M. Squires, Induced-Charge Electrokinetic Phenomena: Theory and Microfluidic Applications. Phys. Rev. Lett., 2004. 92.

8. Ramos, A., et al., Pumping of liquids with travelingwave electroosmosis. Journal of Applied Physics, 2005. 97.

9. Manz, A., et al., Electroosmotic pumping and electrophoretic separations for miniaturized chemical analysis systems. A Manz, C S Effenhauser, N Burggraf, D J Harrison, K Seiler and K Fluri, 1994. 4(4).

10. Urbanski, J.P., et al., Fast ac electro-osmotic micropumps with nonplanar electrodes. Applied Physics Letters, 2006. 89.

11. Islam, N. and J. Wu, Microfluidic Transport by $A C$ Electroosmosis, in International MEMS Conference, I.o.P. Publishing, Editor 2006, Journal of Physics: Conference Series. p. 356-361.

12. Bown, M.R. and C.D. Meinhart, AC electroosmotic flow in a DNA concentrator. Microfluidics and Nanofluidics, 2006. 2(6): p. 513-523.

13. Kemery, P.J., J.K. Steehler, and P.W. Bohn, Electric Field Mediated Transport in Nanometer Diameter Channels. Langmuir, 1998. 14: p. 2884-2889.

14. Prakash, S., et al., Nanofluidics: Systems and Applications. IEEE Sensors Journal, 2008. 8(5): p. 441-450.

15. Sparreboom, W., et al., ION PUMPING IN NANOCHANNELS USING AN ASYMMETRIC ELECTRODE ARRAY, in Twelfth International Conference on Miniaturized Systems for Chemistry and Life Sciences2008: San Diego, CA, United States.

16. Boone, C., et al. Effect of Time Dependent Excitation Signals on Gating in Nanofluidic Channels. in ASME's International Mechanical Engineering Congress and
Exposition (IMECE 2015). 2015. Houston, Texas: ASME.

17. Horiuchi, K. and P. Dutta, Electrokinetic flow control in microfluidic chips using a field-effect transistor. Lab on a Chip, 2006. 6(714-723).

\section{CONTACT}

*S. Prakash, tel: +1-614-688-4045; Prakash. 31@osu.edu 